OPEN ACCESS

Edited by:

Guohua Yuan,

Wuhan University, China

Reviewed by:

Alastair James Sloan,

University of Melbourne, Australia

Anne Poliard,

Université Paris Descartes, France

${ }^{*}$ Correspondence:

Kun Xuan

xuankun@fmmu.edu.cn

${ }^{\dagger}$ These authors have contributed equally to this work

Specialty section:

This article was submitted to Craniofacial Biology and Dental

Research,

a section of the journal

Frontiers in Physiology

Received: 21 January 2021

Accepted: 23 March 2021

Published: 22 April 2021

Citation:

Huang X, Li Z, LiU A, LiU X, Guo H, Wu M, Yang X, Han B and Xuan K (2021) Microenvironment Influences Odontogenic Mesenchymal Stem Cells Mediated

Dental Pulp Regeneration.

Front. Physiol. 12:656588. doi: 10.3389/fphys.2021.656588

\section{Microenvironment Influences Odontogenic Mesenchymal Stem Cells Mediated Dental Pulp Regeneration}

\author{
Xiaoyao Huang ${ }^{1,2,3+}$, Zihan Li1,2,3†, Anqi Liu'1,2,3, Xuemei Liu'1,2,3, Hao Guo 1,2,3, \\ Meiling Wu ${ }^{1,2,3}$, Xiaoxue Yang ${ }^{1,2,3}$, Bing Han ${ }^{1,2,3}$ and Kun Xuan ${ }^{1,2,3 *}$
}

${ }^{1}$ State Key Laboratory of Military Stomatology, Fourth Military Medical University, Xi'an, China, ${ }^{2}$ National Clinical Research Center for Oral Diseases, Fourth Military Medical University, Xi'an, China, ${ }^{3}$ Shaanxi Clinical Research Center for Oral Diseases, Department of Preventive Dentistry, School of Stomatology, Fourth Military Medical University, Xi'an, China

Dental pulp as a source of nutrition for the whole tooth is vulnerable to trauma and bacterial invasion, which causes irreversible pulpitis and pulp necrosis. Dental pulp regeneration is a valuable method of restoring the viability of the dental pulp and even the whole tooth. Odontogenic mesenchymal stem cells (MSCs) residing in the dental pulp environment have been widely used in dental pulp regeneration because of their immense potential to regenerate pulp-like tissue. Furthermore, the regenerative abilities of odontogenic MSCs are easily affected by the microenvironment in which they reside. The natural environment of the dental pulp has been proven to be capable of regulating odontogenic MSC homeostasis, proliferation, and differentiation. Therefore, various approaches have been applied to mimic the natural dental pulp environment to optimize the efficacy of pulp regeneration. In addition, odontogenic MSC aggregates/spheroids similar to the natural dental pulp environment have been shown to regenerate wellorganized dental pulp both in preclinical and clinical trials. In this review, we summarize recent progress in odontogenic MSC-mediated pulp regeneration and focus on the effect of the microenvironment surrounding odontogenic MSCs in the achievement of dental pulp regeneration.

Keywords: odontogenic MSCs, cell aggregate/spheroids, dental pulp regeneration, microenvironment, pulp regeneration approaches

\section{INTRODUCTION}

Being the source of nutrition for the whole tooth, the dental pulp is the residence of a large number of odontogenic mesenchymal stem cells (MSCs), which play an important role in the process of tooth development and injury repair (Lambrichts et al., 2017). However, the dental pulp is prone to traumas and infections, which ultimately lead to the development of irreversible pulpitis or necrosis, because its nutrition is supplied by a tiny apical foramen. The traditional treatment for pulpitis is root canal therapy, which requires removing all the pulp and filling the canals with bioinert synthetic materials. However, it permanently deprives nutrition from teeth, which may increase the friability of the residual tissue of the tooth and arrest the root development of immature permanent teeth ( $\mathrm{Lu}$ et al., 2019). Therefore, maintaining pulp vitality is necessary to save the whole tooth. Subsequently, approaches to restore the pulp viability of immature permanent 
teeth have been established, such as partial pulpotomy and apexification, which make use of pulp cells in the residual dental pulp tissue to repair injured pulp, and made great progress in promoting the root development of immature permanent teeth (Garcia-Godoy and Murray, 2012). Meanwhile, odontogenic MSCs were separated from postnatal dental pulp tissue and developing tooth tissues successively and showed the immense potential to regenerate pulp-like tissues (Gronthos et al., 2000; Miura et al., 2003). Consequently, odontogenic MSCs based dental pulp regeneration has been proposed to maintain teeth vitality. It has made great progress in regenerating a complete dental pulp containing blood vessels, nerves, and newly formed dentin both in ectopic and in situ regeneration (Itoh et al., 2018; Xuan et al., 2018; Meng et al., 2020). Most importantly, it has been demonstrated that exogenous odontogenic MSC transplantation regenerated functional dental pulp, which showed a response to clinical tests similar to those of normal dental pulp (Nakashima and Iohara, 2017; Nakashima et al., 2017; Xuan et al., 2018). The regenerated pulp tissue contained normal structures such as an odontoblast layer, connective tissue, blood vessels, and nervous tissue in histologic examination (Xuan et al., 2018). Therefore, odontogenic MSC based dental pulp regeneration could potentially become a valuable method to restore vital teeth in clinical practice.

The environment of the dental pulp is essential for the regulation of odontogenic MSC homeostasis, proliferation, and differentiation (Smith et al., 2015). When the dental pulp environment is invaded by trauma or bacteria, the MSCs residing in the dental pulp are prone to odontogenic differentiation to repair the dental pulp, but this results in local or total calcification of the pulp tissue rather than the original wellorganized connective tissue (Zhang et al., 2017c). Therefore, rebuilding an environment similar to that of the natural dental pulp is inevitable to induce odontogenic MSCs to regenerate pulp tissue containing normal structures. Various approaches have been applied to mimic the natural dental pulp environment, including its complex mechanical, chemical, and biological properties. The use of cytokines, scaffold materials, and cell aggregates/spheroids is mainstream in current studies and has made great progress both in preclinical studies and clinical trials (Nakashima and Iohara, 2017; Nakashima et al., 2017; Itoh et al., 2018; Xuan et al., 2018; He et al., 2019; Meng et al., 2020). In this review, we summarize recent progress in odontogenic MSC-mediated pulp regeneration, concentrating on the effect of the microenvironment surrounding odontogenic MSCs in the achievement of well-organized functional dental pulp regeneration.

\section{ODONTOGENIC MSCs AND DENTAL PULP REGENERATION}

The dental pulp is a well-organized soft connective tissue in the root canal, with bundles of blood vessels and nerves and a layer of odontoblasts lining along the chamber that can generate the mineralized dentin (Linde, 1985). Regenerating dental pulp containing these well-organized structures is crucial to maintain the function of the dental pulp and to restore the vitality of the whole tooth. Odontogenic MSCs separated from dental pulp and dental apical papilla with immense potential to form pulp-like tissues with blood vessels, nerves, and mineralized dentin was applied for functional dental pulp regeneration. In addition, their ability to regenerate the original well-organized dental pulp is easily affected by the microenvironment in which they are located, and a dental pulp microenvironment that has been changed by infection invasion and root canal preparation is unsuitable for odontogenic MSC-mediated dental pulp regeneration.

\section{MSCs From Dental Pulp Applied in Dental Pulp Regeneration}

The odontogenic MSCs from dental pulp show typical MSC features, including colony formation, expression of specific surface markers, and multi-directional differentiation (Morsczeck and Reichert, 2018). Under physiological conditions, these MSCs reside in the surrounding neurovascular bundle in the dental pulp and act as a reservoir for stem cells capable of differentiating into the various types of cells required for dental pulp maintenance and repair (Zhao et al., 2014). The odontogenic MSCs separated from dental pulp were demonstrated to form blood vessels, nerves, and mineralized dentin, which are regarded as indispensable for functional dental pulp regeneration (Hilkens et al., 2017; Yang et al., 2019) (Figure 1). Hence, the odontogenic MSCs residing in the dental pulp were applied in dental pulp regeneration.

Dental pulp stem cells (DPSCs), the first odontogenic MSCs isolated from dental pulp, have been shown to have the capacity to form mineralized tissue, blood vessels, and nerve tissues. It has been shown that DPSCs can form dentin/pulp-like structures in ectopic transplantation with hydroxyapatite/tricalcium phosphate (Gronthos et al., 2000). In addition, DPSCs have also been shown to be effective in regenerating functional dental pulp with nerves, vasculature, and newly formed dentin in in situ pulp regeneration (Iohara et al., 2014; Nakashima et al., 2017). Stem cells from human exfoliated teeth (SHED), another type of stem cell from dental pulp, were isolated and showed a higher proliferation rate and a higher number of population doublings than DPSCs (Miura et al., 2003). SHED also showed the capacity to differentiate into odontoblasts, generate dentin, and form blood vessels and nerve tissues (Miura et al., 2003; Xuan et al., 2018), and were able to regenerate pulp-like tissues with odontoblasts capable of generating new tubular dentin after being injected into the roots of human premolars with scaffolds (Puramatrix $^{\mathrm{TM}}$ or rhCollagen) in ectopic transplantation experiments (Rosa et al., 2013). Furthermore, SHED has been reported to regenerate functional dental pulp with blood vessels, sensory nerves, and the odontoblast layer in injured immature permanent teeth (Xuan et al., 2018).

\section{Other Odontogenic MSCs in Dental Pulp Regeneration}

In addition to odontogenic MSCs from dental pulp, odontogenic MSCs also include MSCs separated from periodontal tissue 


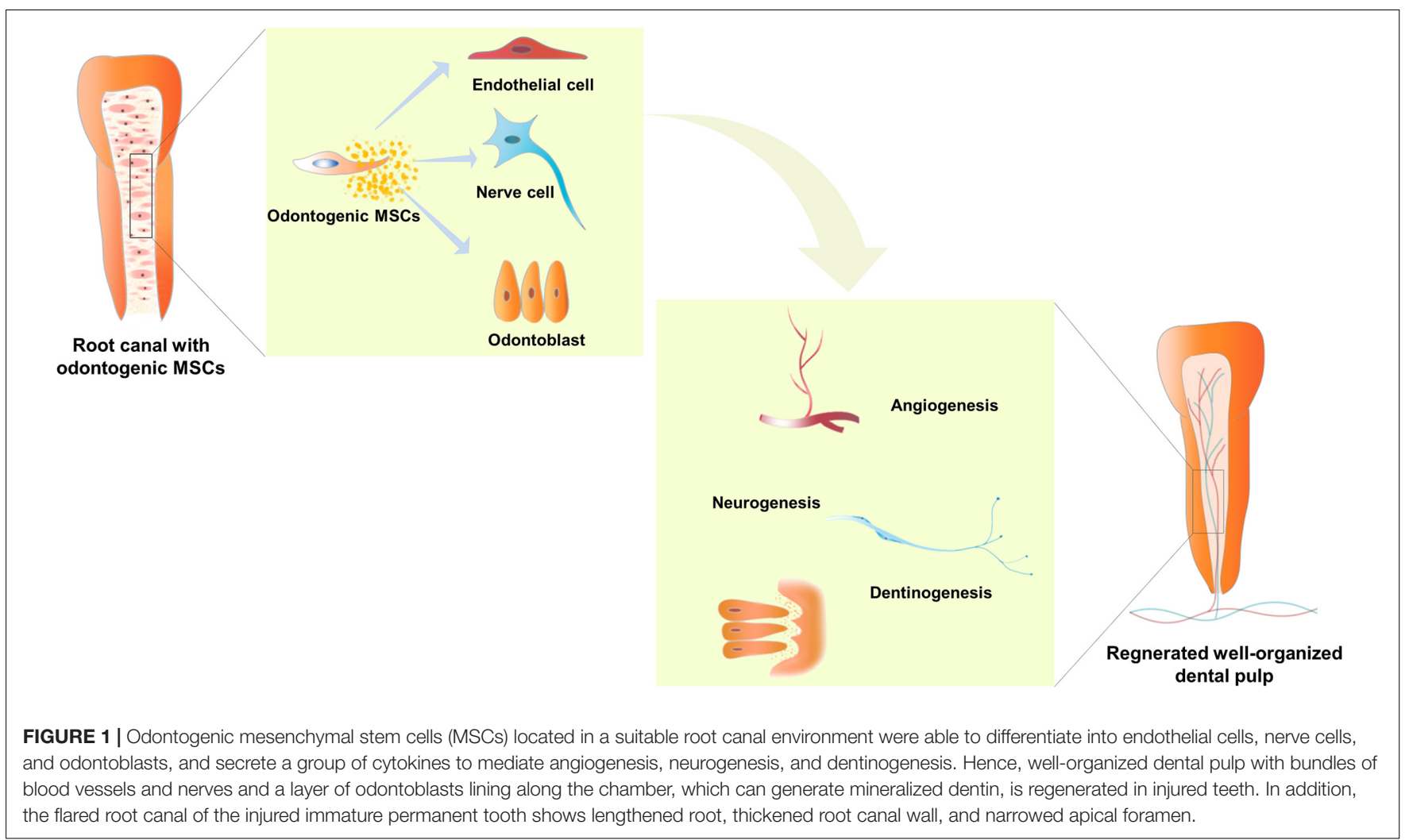

and developing tooth tissues such as the apical papilla and dental follicle. According to research, the odontogenic MSCs from periodontal tissue and dental follicle mainly repair and regenerate periodontal tissue because of the capacity to generate a cementum/periodontal ligament-like structure (Seo et al., 2004). However, stem cells from the apical papilla (SCAPs) isolated from the apical papilla, which was confirmed to develop dental pulp tissue in the early stage of tooth development, were also applied in dental pulp regeneration, and they showed high proliferative potential and were more likely to differentiate into odontoblasts than DPSCs (Sonoyama et al., 2008). It has been demonstrated that SCAPs have a dramatic ability to form dentin, blood vessels, and nerve tissue (Sonoyama et al., 2008; Hilkens et al., 2017), and can regenerate dental pulp with well-established vascularity and a layer of odontoblast-like cells that are able to form dentin-like tissue in ectopic transplantation experiments (Na et al., 2016). However, SCAPs are hardly widely used because they are derived from developing teeth that are scarcely discarded in clinical practice. In general, DPSCs and SHED separated from dental pulp are common odontogenic MSCs for dental pulp regeneration because of their biological properties and extensive sources.

\section{Pulp Regeneration Ability of Odontogenic MSCs Impaired by the Damaged Pulp Microenvironment}

The natural dental pulp tissue is vulnerable to bacteria and traumas, which cause cell death among odontoblasts and other cells in the dental pulp and distort the collagen arrangement in the pulp matrix. Then, the odontogenic MSCs residing in the dental pulp will be subsequently recruited to the injured site by a cascade of signal molecules to differentiate into odontoblasts and form reparative dentin, which is a type of calcified tissue diffused in the root canal rather than the original soft connective tissue (Colombo et al., 2014; Zhang et al., 2017c). If the infection persists, the dental pulp is prone to develop irreversible pulpitis or necrosis, that is, the natural dental pulp microenvironment is damaged, and bacteria, necrotic tissue, toxins, and dead cells, etc. will remain in the root canal, which are unfavorable for maintaining the biological properties of odontogenic MSCs to regenerate dental pulp. Even when the root canal was cleaned before pulp regeneration, the bacteria and inflammation cannot be cleaned due to the complexity of the root canal system.

Research on dental pulp regeneration in animal models has shown that residual bacteria remain in the root canal after traditional root canal preparation and disinfection, and empty space or necrotic tissue was found between residual bacteria and revitalized tissue (Verma et al., 2017). Meanwhile, lipopolysaccharide (LPS), a glycolipid in the outer membrane of gram-negative bacteria, was reported to increase the levels of inflammatory mediators, such as IL- $1 \beta$ and TNF- $\alpha$ (Yuan et al., 2018), and interfere with dentinogenesis of DPSCs (Hozhabri et al., 2015). In addition, DPSCs with repeated LPS stimulation induced DNA double-strand breaks and DNA damage responses, which had a significant influence on cell proliferation and apoptosis (Feng et al., 2014; Huang et al., 2018). Moreover, the root canal is under an ischemic condition when dental pulp is 
removed, which means there is no oxygen or nutrients in the root canal. Agata et al. (2008) have shown that ischemia has a significantly negative influence on dental pulp cell survival and differentiation. Similarly, it has been reported that hypoxia decreases cell viability and increases cleaved caspase- 3 and poly ADP-ribose polymerase in human dental pulp cells, indicating that it induces apoptotic cell death in these cells (Park et al., 2018). It has also been shown that cells die in the center of large-sized DPSCs spheroids due to the ischemic environment in the center (Xiao et al., 2014), which is similar to the condition that MSCs confront in the root canal.

Overall, the potential of odontogenic MSCs to regenerate well-organized dental pulp tissue is mediated by the microenvironment in which they reside, and the dental pulp microenvironment damaged by infection, and the prepared root canals with inflammatory and ischemic conditions are unfavorable for odontogenic MSCs to regenerate original well-organized connective pulp tissue. Optimizing the external environment of odontogenic MSCs is crucial for promoting the effect of pulp regeneration.

\section{MIMIC THE NATURAL PULP MICROENVIRONMENT TO IMPROVE THE PULP REGENERATION EFFICACY}

The natural dental pulp microenvironment is crucial for the maintenance of a stem cell phenotype that is suitable for downstream pulp regeneration applications (Smith et al., 2015). Therefore, it is feasible to mimic the physiological natural pulp microenvironment to maintain the regeneration ability of odontogenic MSCs when the environment is changed due to bacterial invasion and root canal preparation. Based on this, researchers have found some methods to improve the efficacy of dental pulp regeneration by maintaining the regeneration ability of odontogenic MSCs that reside in the inflammatory and ischemic microenvironment.

\section{Cytokine Application}

Cooper et al. (2014) have reviewed that the body can produce various types of cytokines that can promote the migration, proliferation, and differentiation of MSCs to maintain dental pulp homeostasis under physiological conditions. It is widely believed that cytokines have the potential to recruit endogenous MSCs to repair damaged tissue, which is defined as cell homing and applied in dental pulp regeneration (Kim et al., 2010).

Stromal cell-derived factor 1 (SDF-1), a member of the CXC chemokine subfamily, was reported to regenerate pulp-like tissue in pulpectomy mature teeth of dogs (Iohara et al., 2011). And researchers have found that SDF-1 can recruit odontogenic MSCs via SDF-1/C-X-C chemokine receptor type 4 (CXCR4) pathway (Yang et al., 2016; Xiao et al., 2019); mediate mineralization tissue formation by activation of Smads and Erk (Liu et al., 2015; Xiao et al., 2019) and promote vascularization through autophagy (Yang et al., 2015). In addition, granulocyte-colony stimulating factor (G-CSF) which was allowed for clinical application was also reported to have migratory efficacy on pulp stem cells (Iohara et al., 2008) and was shown to regenerate total pulp with pulp stem cells in the pulpectomized teeth of dogs (Iohara et al., 2013). Murakami et al. (2013) found that DPSCs with migratory response to G-CSF (MDPSCs) showed enhanced migration and immunomodulatory abilities and expressed higher stem cell markers Oct3/4, Nanog, Rex1, GDF3 compared to DPSCs, which means a better regeneration ability. A pilot clinical study demonstrated that MDPSCs transplantation showed excellent clinical efficacy in patients with Pulpitis. In addition, multiple growth factors were applied to recruit odontogenic MSCs and promote nerve, blood vessels, and mineralized tissue formation. Nerve growth factor (NGF) plays a role in attracting nerve fiber growth into the root canal and has been reported to mediate the proliferative differentiation and survival of odontogenic MSCs (Mitsiadis et al., 2017) and promote mineralized tissue formation (Xiao et al., 2018).

Fibroblast growth factor (FGF) expressed in enamel knots during primary dentinogenesis has been reported to induce dentin regeneration on amputated pulp (Ishimatsu et al., 2009). Platelet-derived growth factor (PDGF) and vascular endothelial growth factor (VEGF) play a major role in angiogenesis and were shown to generate highly vascularized dental pulp-like connective tissue (Kim et al., 2010; Zhang et al., 2017a). Furthermore, stem cell factor (Pan et al., 2013), tumor necrosis factor-a (TNF- $\alpha$ ) (Shi et al., 2017), interferon- $\gamma$ (IFN- $\gamma$ ) (He et al., 2017b), and BMP (Kim et al., 2010) have also been reported to promote dental pulp regeneration by increasing odontogenic MSCs migration and differentiation. To achieve satisfactory regeneration efficacy, these cytokines are usually applied in combination with other cytokines, scaffolds, and MSCs. It has been reported that combinations of SDF-1, bFGF, and BMP7 in collagen scaffolds were efficient in regenerating pulp-like tissues in endodontically treated human root canals subcutaneously implanted in rats (Suzuki et al., 2011).

\section{Scaffold Material Application}

It has been proven that signals from extracellular matrix (ECM) microenvironments significantly affect stem cell migration, proliferation, and differentiation (Cosgrove et al., 2016). Scaffold materials act as odontogenic MSC ECM in the regeneration of dental pulp, and changes in their mechanical properties, composition, and structure will affect the biological properties of MSCs (Shafiq et al., 2015; Rahman et al., 2018; Huang et al., 2019). As recently reviewed by Moussa and Aparicio (2019), the scaffold materials applied for dental pulp regeneration mainly include: (1) naturally derived polymeric scaffolds like collagen, fibrin, decellularized dental pulp tissue (Alqahtani et al., 2018; Bakhtiar et al., 2020), and treated dentin matrix (TDM) (Yang et al., 2012; Meng et al., 2020); (2) synthetically engineered polymeric scaffolds such as polylactic acid, polyglycolic acid, and ceramic scaffolds; and (3) composite scaffolds that balance the advantages and disadvantages of individual material, improving the overall material performance (Moussa and Aparicio, 2019).

These scaffold materials rebuild a suitable environment for odontogenic MSCs to regenerate dental pulp tissue by changing mechanical properties, composition, and structure and combining them with cytokines and other scaffold materials. 
In order to prevent the growth of residual endodontic bacteria, chitosan was applied as a scaffold material (Ducret et al., 2019). In addition, the nanofibrous engineered matrix with fibrous topography similar to dental pulp matrix was proved to induce odontoblastic differentiation of DPSCs through Wnt/ $\beta$-catenin signaling (Rahman et al., 2018). Remarkably, naturally derived materials showed a better capability of maintaining dental pulp stem cell viability and forming pulp-like tissue compared with all synthetic materials. Decellularized dental pulp tissue containing collagen type I, dentin matrix protein 1, dentin sialoprotein, Von Willebrand factor, TGF- $\beta$, VEGF, and bFGF was showed an increased induction of DPSCs proliferation, migration, and multidirectional differentiation (Alqahtani et al., 2018; Li et al., 2020). And more convincing, the decellularized tooth buds seeded with stem cells were able to regenerate well-developed teeth after implanted into the jawbone of mini-pigs (Zhang et al., 2017b). Furthermore, TDM comprised of hydroxyapatite and ECM was reported to be the reservoir of bioactive protein necessary for dentinogenesis (Li et al., 2011). Thus, researchers found that TMD can release dentinogenic factors and growth factors to improve the attachment, growth, and viability of odontogenic MSCs and induce DPSCs to form dentin pulplike tissue (Meng et al., 2020). This proves that similar scaffold materials mimic the natural pulp environment and that the more similar they are, the better the dental pulp regeneration.

\section{Extracellular Vesicles Application}

Extracellular vesicles (EVs) are particles secreted from cells and composed of a lipid bilayer carrying bioactive molecules like mRNA, micoRNA, and cytokines, etc. (Zhang et al., 2020a). The biological function of EVs is dominated by the contents of EVs and varied according to the tissue they are derived from. Thus, EVs generated from the dental pulp cells residing in the relatively closed root canal were demonstrated to have advantageous proangiogenic, antiapoptotic, anti-inflammatory, and immunomodulatory abilities when applied in tissue regeneration (Jarmalavičiūtè et al., 2015; Pivoraitè et al., 2015; Xian et al., 2018; Hu et al., 2019).

Recently, odontogenic MSCs derived EVs have drawn attention in the field of dental pulp regeneration. Huang et al. (2016) demonstrated that DPSC derived EVs mediated the odontogenic differentiation of DPSCs through the P38 MAPK pathway and regenerated pulp-like tissue when composited with collagen membrane in a tooth root slice model. Besides, Zhang et al. (2020a) proved that EVs from DPSCs can promote angiogenesis and induce collagen deposition along neovasculature in an injectable hydrogel in vitro, which indicates the initiation of pulp-like tissue formation (Zhang et al., 2020a). More importantly, Zhang et al. (2020b) presented that EVs generated by Hertwig's epithelial root sheath cells which exist in the developing period of teeth can trigger lineage-specific differentiation of dental papilla cells and regenerate dentinpulp like tissue which is composed of dentin-like hard tissue and soft tissue containing blood vessels and neurons (Zhang et al., 2020b). In general, EVs derived from dental pulp cells can provide proper microenvironments that mimic the process of angiogenesis, dentin formation, and epithelia-mesenchyme interactions in tooth development, which means that EVs from dental pulp are a favorable choice for dental pulp regeneration.

Since the contents of EVs are abundant and varied according to the environment, further research should be carried out to optimize the contents of EVs, making them more suitable for dental pulp regeneration. In addition, suitable scaffold materials must be picked out to maintain the biologic function of EVs and facilitate them applied into root canals, and in situ dental pulp regeneration experiments and clinical research are required to verify the efficacy and safety of EVs in dental pulp regeneration.

\section{Cell Aggregates/Spheroids Application}

Cell aggregates and spheroids have been demonstrated to consist of high-density stem cells with a self-produced, tissue-specific ECM, multiple cytokines, and large amounts of extracellular vesicles (Tsai et al., 2015; Sjöqvist et al., 2019; Sui et al., 2019b). Besides, cell aggregates/spheroids were reported to show enhanced abilities anti-inflammatory and anti-ischemic abilities compared to regular cultured cells (Tsai et al., 2015; Sukho et al., 2018; Cheng et al., 2020), which means cell aggregates/spheroids are suitable for the inflammatory and ischemic environment of the root canal.

Cell aggregates/spheroids have been proven to promote the anti-inflammatory phenotype differentiation of macrophages and express more anti-inflammatory signaling molecules including CXCR4, prostaglandin E2 (PGE-2), and interleukin 6 (IL-6) when compared to regular cultured cells, which means cells in cell aggregates/spheroids have better cell migration and anti-inflammatory abilities (Tsai et al., 2015; Sukho et al., 2018). Other studies have shown that the center of cell aggregates/spheroids is in an ischemic and hypoxia condition, thus cell aggregates/spheroids contain more trophic factors and pro-angiogenic factors (like VEGF, FGF-F, and HGF) and have great pro-angiogenesis capabilities (Bhang et al., 2011; Zhang et al., 2012; Cheng et al., 2020). Based on this, cell aggregates/spheroids have been proven to be the reservoir of the cytokines that are essential for odontogenic MSCs to migrate, proliferate, differentiate and regenerate dental pulp. Furthermore, compared to regular cultured cells, cell aggregates express more ECM protein like COLI, integrin $\beta 1$, and fibronectin which are crucial for cell signaling transmission and biological property maintenance (Yang et al., 2012; Yavropoulou and Yovos, 2016). Studies have shown that DPSC aggregate-derived ECM (DPM) preserved the important fibrous portions of dental pulp tissue-derived ECM and showed a similar 3D structure of dental pulp, and the DPM provides a microenvironment to balance the replication and mineralization of the behaviors of DPSCs in a way similar to a natural dental pulp microenvironment (Zhang et al., 2017c). The comparison of gene expression among SCAP cell aggregates, SCAPs, and apical papilla tissues, which generate pulp and dentin during tooth development, showed that SCAP cell aggregates can recover some gene expression in the apical papilla niche compared to SCAPs (Diao et al., 2017).

Therefore, odontogenic MSC aggregates/spheroids are considered fantastic martial to realize dental pulp regeneration. It has been shown that after implant a human tooth root canal which was filled with DPSC spheroids into immunodeficient mice 
subcutaneously, pulp-like tissues with blood vessel ingrowths were observed in the human root canal (Itoh et al., 2018). Similarly, implanted SCAP aggregates into immunodeficient mice with human treated dentin matrix fragments, the root space was found to be filled completely with dental pulp-like tissue with well-organized vascularity and a continuous layer of newly formed dentin-like tissue along the existing dentin (Na et al., 2016). Furthermore, our clinical trial found that the implantation of SHED aggregates induces the regeneration of functional pulp tissue with blood vessels and sensory nerves in immature permanent teeth with pulp necrosis after dental trauma (Xuan et al., 2018).

\section{PROGRESS OF DENTAL PULP REGENERATION}

Dental pulp regeneration methods, including pulp revascularization, cytokine combined with scaffold transplantation, and exogenous odontogenic MSC transplantation were put into practice in situ dental pulp regeneration in animals or patients, based on approaches put forward to optimize the surrounding microenvironment of odontogenic MSCs involved in the pulp regeneration process. The first two methods are claimed to recruit endogenous MSCs by cytokines and without the application of exogenous odontogenic MSCs, while the latter method applies exogenous odontogenic MSCs alone or with cytokines and scaffold materials and has demonstrated dramatic efficacy of functional dental pulp regeneration both in preclinical animal studies and clinical trials (Figure 2).

\section{Pulp Revascularization}

Pulp revascularization has been practiced clinically in the past decade (Wigler et al., 2013; Gaviño Orduña et al., 2020). In this method, an optimized microenvironment was built in the disinfected root canal system with endodontic instrumentinduced blood clots (Yang et al., 2016). It is believed that oxygen, nutrients, and endogenous cytokines, and MSCs are brought into the root canal by blood clots (He et al., 2017a), and there is no need for exogenous cytokines or stem cells.

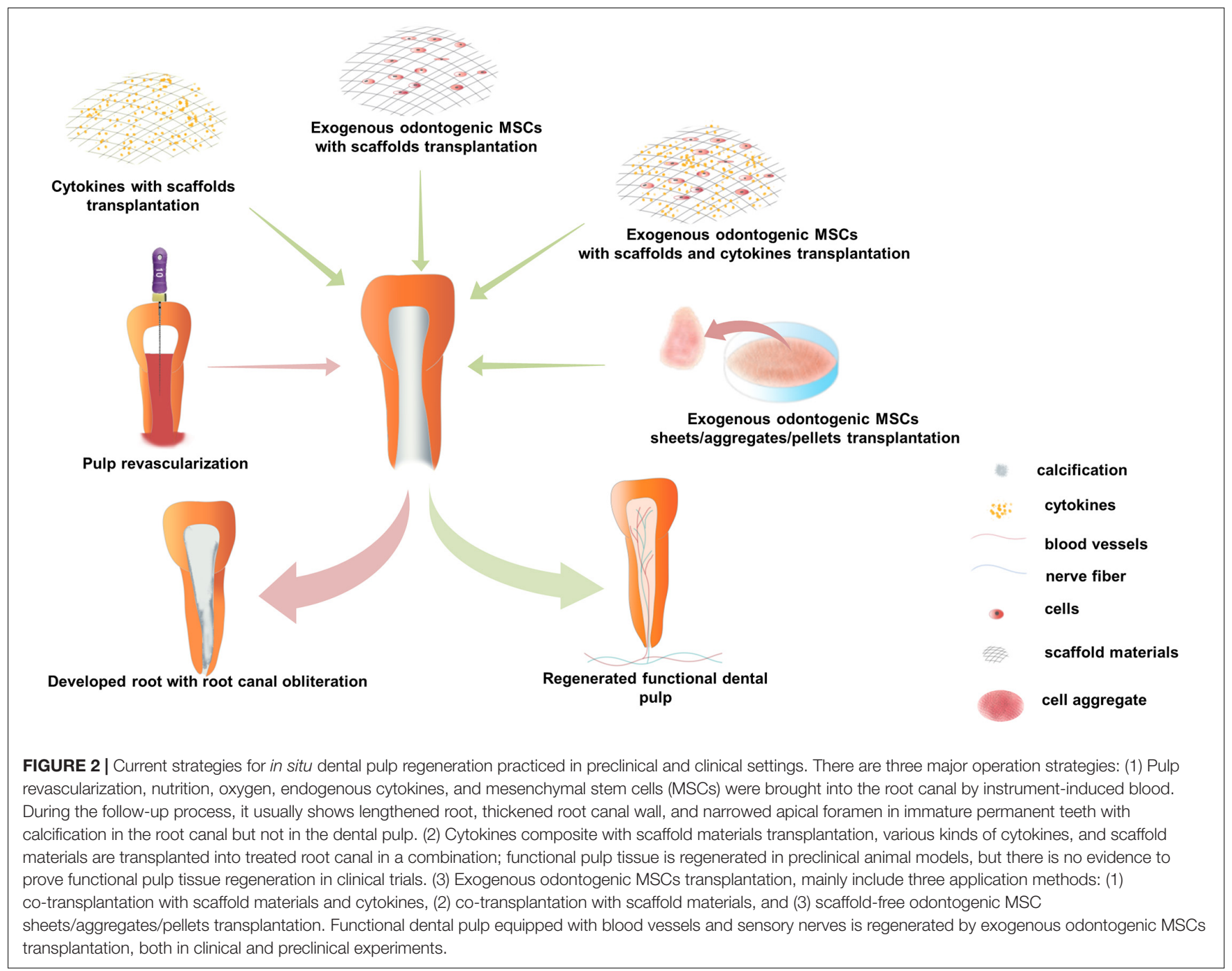


Some cases of pulp revascularization have shown sensitivity to cold or electric stimuli in the clinical examination of pulp vitality (He et al., 2017a), while some cases showed no response to thermal or electric stimuli during long-term follow-up. Radiographic examination revealed that the root was lengthened, the wall of the root canal was thickened, and the apical foramen was narrowed in some cases of immature permanent teeth (Nagata et al., 2015; Peng et al., 2017). However, histological studies have shown that most of the tissues generated in pulp revascularization cases were finally turned into non-pulp-like tissues including cementum, periodontal, and bone-like tissues because endogenous MSCs and cytokines brought in by blood clots are uncontrollable and MSCs from the periodontal ligament and bone marrow might be brought into root canals (Peng et al., 2017) (Figure 2). Therefore, the formation of pulplike tissues requires further studies, and multiple exogenous cytokines and odontogenic MSCs should be applied in dental pulp regeneration.

\section{Cytokines Combined With Scaffold Transplantation}

Cytokines can induce odontogenic MSCs to migrate, proliferate, and differentiate, while scaffold materials can not only influence the biological properties of odontogenic MSCs but also provide attachment for cells (Kim et al., 2010; Moussa and Aparicio, 2019). Therefore, exogenous cytokines are usually transplanted with scaffold materials, creating an environment to induce endogenous stem cells to regenerate dental pulp tissue (Figure 2). It has been proven that the delivery of bFGF, VEGF, or PDGF with a basal set of NGF and BMP7 in collagen scaffolds regenerated dental-pulp-like tissue in the entire root canal in an ectopic transplantation animal model (Kim et al., 2010). Similar studies have shown that a combination of Wnt3a, BMP7, and collagen gel delivered into the root canal of mini-pigs yielded pulp-dentin-like structures with obvious dentinal tubules (He et al., 2019). Furthermore, many bloodderived scaffold materials containing rich growth factors are used in clinical pulp therapy, such as platelet-rich plasma (PRP), concentrated growth factors, and platelet-rich fibrin, and showed a much better influence on cell proliferation, viability, apoptosis, and mineralization of human dental pulp cells than traditional materials such as $\mathrm{Ca}(\mathrm{OH})_{2}$, mineral trioxide aggregate, and iRoot BP (Dou et al., 2020). Moreover, Jadhav et al. (2012) proved that PRP showed a better treatment effect in periapical healing, apical closure, and dentinal wall thickening compared to blood clots in regenerative endodontic tissue. However, histological evidence showed that there was no difference in the average percentage of apical closure, new tissue formation, and pulp-like tissue formation between PRP and blood clots in regenerative endodontic treatment of animal models (Zhang et al., 2014).

It is claimed that the regenerated pulp-like tissue in the root canal and the better treatment effect of blood-derived scaffold materials were due to the endogenous stem cells recruited by exogenous cytokines (Kim et al., 2010; Suzuki et al., 2011; Zhujiang and Kim, 2016). However, there is no direct evidence to prove that the pulp-like tissue was regenerated by recruited endogenous MSCs, and no clinical trials or research to confirm the functional in situ pulp regeneration with exogenous cell-free approaches (Sui et al., 2019a), while clinical trials have achieved functional dental pulp regeneration in injured immature permanent teeth and permanent teeth with pulpitis by exogenous odontogenic MSC transplantation (Nakashima and Iohara, 2017; Nakashima et al., 2017; Xuan et al., 2018). In summary, exogenous stem cell implantation can be considered a promising approach for dental pulp regeneration.

\section{Exogenous Odontogenic MSCs Transplantation}

The application of exogenous odontogenic MSCs in in situ pulp regeneration has been practiced in preclinical animal models and clinical patients. Since the external microenvironment is crucial for the maintenance of the biological properties of odontogenic MSCs (Smith et al., 2015) to promote their capacity to regenerate dental pulp tissue, various methods have been applied to maintain the external microenvironment of transplanted odontogenic MSCs, such as scaffolds and cytokines, and applied in the form of cell aggregates/spheroids.

Exogenous odontogenic MSCs transplanted with scaffold materials and cytokines were proven feasible both in preclinical and clinical trials. Studies on animals have shown that side populations of DPSCs transplanted with cytokines (G-CSF and SDF-1) into the treated root canal of dogs regenerated pulp in situ, including nerves and vasculature, and new dentin deposition along the dentinal wall (Iohara et al., 2011, 2014) (Figure 2). Furthermore, clinical trials have also proved that autologous DPSC subsets transplanted with G-CSF and atelocollagen into pulpectomized teeth regenerated pulp tissue, which showed a robust positive response to the electric pulp test (EPT), showed the similar signal intensity of magnetic resonance imaging to that of the normal dental pulp, demonstrated functional dentin formation in conebeam computed tomography (СВCT) test, and showed no adverse events or toxicity in the clinical and laboratory evaluations (Nakashima and Iohara, 2017; Nakashima et al., 2017). Meanwhile, transplantation of exogenous odontogenic MSC aggregates was also proven to be effective in preclinical studies and clinical trials. It has been demonstrated that the transplantation of the side populations of DPSCs cell aggregates into an in vivo model of amputated pulp and pulp tissue with capillaries and nerves was regenerated (Iohara et al., 2009). Furthermore, our recent study of mini-pigs demonstrated that the transplantation of pig DPSC aggregates into endodontically treated pig teeth regenerated functional dental pulp with blood vessels and nerves (Figure 2). Meanwhile, we carried out a randomized clinical trial with autologous SHED aggregate transplantation in patients with tooth trauma. Thirty-six patients with pulp necrosis after dental trauma were included, with 26 patients in the SHED aggregate transplantation group and 10 patients in the traditional apexification treatment group. After a 12-month follow-up, the SHED aggregate transplantation group showed regeneration of well-organized pulp tissue with blood 
vessels and sensory nerve ingrowth histologically, and clinical examination showed robust positive results in the EPT, increased vascular formation in laser Doppler flowmetry, and increased length of the root and closed apical foramen in CBCT. Furthermore, there were no adverse events at the 24-month follow-up (Xuan et al., 2018). These studies suggest that exogenous odontogenic MSC implantation may be an effective approach to regenerate functional dental pulp, and the application of odontogenic MSC aggregates may be a promising method for future regenerative endodontics in clinical settings.

\section{DISCUSSION}

The efficacy of dental pulp regeneration depends on the biological properties of the odontogenic MSCs involved in the regeneration process, and the natural dental pulp microenvironment of odontogenic MSCs is essential to regulate their homeostasis, proliferation, and differentiation; thus, mimicking the natural pulp microenvironment is the key to realizing pulp regeneration.

By summarizing recent achievements in pulp regeneration, we found that exogenous odontogenic MSC transplantation has made dramatic progress both in preclinical research and clinical trials (Nakashima and Iohara, 2017; Nakashima et al., 2017; Xuan et al., 2018). In addition, odontogenic MSC aggregates show immense potential to regenerate well-organized pulp (Iohara et al., 2009; Na et al., 2016; Itoh et al., 2018; Xuan et al., 2018; Meng et al., 2020). This is likely because the ECM of odontogenic MSCs was similar to that of dental pulp tissue-derived ECM (Zhang et al., 2017c). Furthermore, the dental pulp regenerated by the transplantation of cell aggregates into treated root canals is similar to the process of pulp-dentin complex development. Before the regeneration procedure, the root canal was routinely treated with ethylene diamine tetra-acetic acid, which was been proven to expose the dentin tubules, loosen the intertubular and peritubular dentin, and promote the release of dentin growth factors. These structure and growth factors not only play important roles in inducing MSC proliferation and differentiation but also offer a scaffold to form dentin tissues and control mineralization during dentin regeneration ( $\mathrm{Li}$ et al., 2011). Cell aggregates have been shown to preserve the normal cellular junctions and endogenous ECM similar to their natural microenvironment, and to mimic the mechanical, chemical, and biological properties of the natural microenvironment (Diao et al., 2017). When cell aggregates were implanted into the root canal, the interaction between cell aggregates and the treated dentin is similar to that between the newly formed dentin and the apical papilla, which is the aggregate of odontogenic MSCs in the early

\section{REFERENCES}

Agata, H., Kagami, H., Watanabe, N., and Ueda, M. (2008). Effect of ischemic culture conditions on the survival and differentiation of porcine dental stage of tooth development. Therefore, we hypothesize it may be possible that transplantation of cell aggregates into treated root canals simulated the microenvironment of the pulp-dentin complex development and initiated the process of pulp-dentin complex development. Meanwhile, recent research has demonstrated that Alx3, a transcription factor highly expressed in developing teeth, regenerated parenchymal and stromal tissue of the tooth. Wnt3a, as Alx3's direct target delivered in endodontically prepared root canals, was shown to regenerate both the parenchyma and stroma in adult teeth (He et al., 2019). From this, we can see that signals from the development microenvironment of teeth play a pivotal role in dental pulp regeneration. Therefore, mimicking the development of the microenvironment of the pulp-dentin complex will probably become a feasible and effective approach in dental pulp regeneration.

\section{CONCLUSION}

In conclusion, the microenvironment surrounding odontogenic MSCs can easily influence the biological properties of odontogenic MSCs and affect the efficacy of dental pulp regeneration. Methods mimicking the natural dental pulp microenvironment are effective in regenerating well-organized functional dental pulp by maintaining homeostasis, proliferation, and differentiation of the odontogenic MSCs. Moreover, simulating the development of the microenvironment of the pulp-dentin complex may be a more effective approach to regenerate dental pulp.

\section{AUTHOR CONTRIBUTIONS}

$\mathrm{XH}, \mathrm{ZL}$, and AL conceptualized the review. XH, HG, and XL prepared the figures. $\mathrm{ZL}$ and $\mathrm{XH}$ revised the manuscript. $\mathrm{KX}$ supervised the work. All authors contributed to writing the manuscript and approved the submitted version.

\section{FUNDING}

This work was supported by the National Natural Science Foundation of China (Grant Nos. 82071075 and 81900957).

\section{ACKNOWLEDGMENTS}

Thanks to KX for the choice of authors and for bringing them together to complete this manuscript. $\mathrm{XH}$ would like to thank $\mathrm{KX}$, $\mathrm{AL}, \mathrm{XL}, \mathrm{HG}$, and MW for their guidance and encouragement.

pulp-derived cells. Differentiation 76, 981-993. doi: 10.1111/j.1432-0436.2008 00282.x

Alqahtani, Q., Zaky, S. H., Patil, A., Beniash, E., Ray, H., and Sfeir, C. (2018). Decellularized swine dental pulp tissue for regenerative root 
canal therapy. J. Dent. Res. 97, 1460-1467. doi: 10.1177/00220345187 85124

Bakhtiar, H., Pezeshki-Modaress, M., Kiaipour, Z., Shafiee, M., Ellini, M. R., Mazidi, A., et al. (2020). Pulp ECM-derived macroporous scaffolds for stimulation of dental-pulp regeneration process. Dent. Mater. 36, 76-87. doi: 10.1016/j.dental. 2019.10.011

Bhang, S. H., Cho, S. W., La, W. G., Lee, T. J., Yang, H. S., Sun, A. Y., et al. (2011). Angiogenesis in ischemic tissue produced by spheroid grafting of human adipose-derived stromal cells. Biomaterials 32, 2734-2747. doi: 10.1016/ j.biomaterials.2010.12.035

Cheng, N. C., Tu, Y. K., Lee, N. H., and Young, T. H. (2020). Influence of human platelet lysate on extracellular matrix deposition and cellular characteristics in adipose-derived stem cell sheets. Front. Cell Dev. Biol. 8:558354. doi: 10.3389/ fcell.2020.558354

Colombo, J. S., Moore, A. N., Hartgerink, J. D., and D'souza, R. N. (2014). Scaffolds to control inflammation and facilitate dental pulp regeneration. J. Endod. 40, S6-S12. doi: 10.1016/j.joen.2014.01.019

Cooper, P. R., Holder, M. J., and Smith, A. J. (2014). Inflammation and regeneration in the dentin-pulp complex: a double-edged sword. J. Endod. 40, S46-S51. doi: 10.1016/j.joen.2014.01.021

Cosgrove, B. D., Mui, K. L., Driscoll, T. P., Caliari, S. R., Mehta, K. D., Assoian, R. K., et al. (2016). N-cadherin adhesive interactions modulate matrix mechanosensing and fate commitment of mesenchymal stem cells. Nat. Mater. 15, 1297-1306. doi: 10.1038/nmat4725

Diao, S., Lin, X., Wang, L., Dong, R., Du, J., Yang, D., et al. (2017). Analysis of gene expression profiles between apical papilla tissues, stem cells from apical papilla and cell sheet to identify the key modulators in MSCs niche. Cell Prolif. 50:e12337. doi: 10.1111/cpr.12337

Dou, L., Yan, Q., and Yang, D. (2020). Effect of five dental pulp capping agents on cell proliferation, viability, apoptosis and mineralization of human dental pulp cells. Exp. Ther. Med. 19, 2377-2383. doi: 10.3892/etm.2020. 8444

Ducret, M., Montembault, A., Josse, J., Pasdeloup, M., Celle, A., Benchrih, R., et al. (2019). Design and characterization of a chitosan-enriched fibrin hydrogel for human dental pulp regeneration. Dent. Mater. 35, 523-533. doi: 10.1016/j. dental.2019.01.018

Feng, X., Feng, G., Xing, J., Shen, B., Tan, W., Huang, D., et al. (2014). Repeated lipopolysaccharide stimulation promotes cellular senescence in human dental pulp stem cells (DPSCs). Cell Tissue Res. 356, 369-380. doi: 10.1007/s00441014-1799-7

Garcia-Godoy, F., and Murray, P. E. (2012). Recommendations for using regenerative endodontic procedures in permanent immature traumatized teeth. Dent. Traumatol. 28, 33-41. doi: 10.1111/j.1600-9657.2011.01044.x

Gaviño Orduña, J. F., García García, M., Dominguez, P., Caviedes Bucheli, J., Martin Biedma, B., Abella Sans, F., et al. (2020). Successful pulp revascularization of an autotransplantated mature premolar with fragile fracture apicoectomy and plasma rich in growth factors: a 3-year follow-up. Int. Endod. J. 53, 421-433. doi: 10.1111/iej.13230

Gronthos, S., Mankani, M., Brahim, J., Robey, P. G., and Shi, S. (2000). Postnatal human dental pulp stem cells (DPSCs) in vitro and in vivo. Proc. Natl. Acad. Sci. U.S.A. 97, 13625-13630. doi: 10.1073/pnas.240309797

He, L., Kim, S. G., Gong, Q., Zhong, J., Wang, S., Zhou, X., et al. (2017a). Regenerative endodontics for adult patients. J. Endod. 43, S57-S64. doi: 10. 1016/j.joen.2017.06.012

He, L., Zhou, J., Chen, M., Lin, C. S., Kim, S. G., Zhou, Y., et al. (2019). Parenchymal and stromal tissue regeneration of tooth organ by pivotal signals reinstated in decellularized matrix. Nat. Mater. 18, 627-637. doi: 10.1038/s41563-0190368-6

He, X., Jiang, W., Luo, Z., Qu, T., Wang, Z., Liu, N., et al. (2017b). IFN- $\gamma$ regulates human dental pulp stem cells behavior via NF-кB and MAPK signaling. Sci. Rep. 7:40681. doi: 10.1038/srep40681

Hilkens, P., Bronckaers, A., Ratajczak, J., Gervois, P., Wolfs, E., and Lambrichts, I. (2017). The angiogenic potential of DPSCs and SCAPs in an In Vivo model of dental pulp regeneration. Stem Cells Int. 2017:2582080. doi: 10.1155/2017/ 2582080

Hozhabri, N. S., Benson, M. D., Vu, M. D., Patel, R. H., Martinez, R. M., Nakhaie, F. N., et al. (2015). Decreasing NF- $\mathrm{B}$ expression enhances odontoblastic differentiation and collagen expression in dental pulp stem cells exposed to inflammatory cytokines. PLoS One 10:e0113334. doi: 10.1371/journal.pone. 0113334

Hu, X., Zhong, Y., Kong, Y., Chen, Y., Feng, J., and Zheng, J. (2019). Lineagespecific exosomes promote the odontogenic differentiation of human dental pulp stem cells (DPSCs) through TGF 1 1/smads signaling pathway via transfer of microRNAs. Stem Cell Res. Ther. 10:170. doi: 10.1186/s13287-019-1278-x

Huang, C. C., Narayanan, R., Alapati, S., and Ravindran, S. (2016). Exosomes as biomimetic tools for stem cell differentiation: applications in dental pulp tissue regeneration. Biomaterials 111, 103-115. doi: 10.1016/j.biomaterials.2016. 09.029

Huang, R., Wang, J., Chen, H., Shi, X., Wang, X., Zhu, Y., et al. (2019). The topography of fibrous scaffolds modulates the paracrine function of Ad-MSCs in the regeneration of skin tissues. Biomater. Sci. 7, 4248-4259. doi: 10.1039/ c9bm00939f

Huang, Y., Qiao, W., Wang, X., Gao, Q., Peng, Y., Bian, Z., et al. (2018). Role of Ku70 in the apoptosis of inflamed dental pulp stem cells. Inflamm. Res. 67, 777-788. doi: 10.1007/s00011-018-1167-2

Iohara, K., Imabayashi, K., Ishizaka, R., Watanabe, A., Nabekura, J., Ito, M., et al. (2011). Complete pulp regeneration after pulpectomy by transplantation of CD105+ stem cells with stromal cell-derived factor-1. Tissue Eng. Part A 17, 1911-1920. doi: 10.1089/ten.TEA.2010.0615

Iohara, K., Murakami, M., Nakata, K., and Nakashima, M. (2014). Age-dependent decline in dental pulp regeneration after pulpectomy in dogs. Exp. Gerontol. 52, 39-45. doi: 10.1016/j.exger.2014.01.020

Iohara, K., Murakami, M., Takeuchi, N., Osako, Y., Ito, M., Ishizaka, R., et al. (2013). A novel combinatorial therapy with pulp stem cells and granulocyte colony-stimulating factor for total pulp regeneration. Stem Cells Transl. Med. 2, 521-533. doi: 10.5966/sctm.2012-0132

Iohara, K., Zheng, L., Ito, M., Ishizaka, R., Nakamura, H., Into, T., et al. (2009). Regeneration of dental pulp after pulpotomy by transplantation of CD31(-)/CD146(-) side population cells from a canine tooth. Regen. Med. 4, 377-385. doi: 10.2217/rme.09.5

Iohara, K., Zheng, L., Wake, H., Ito, M., Nabekura, J., Wakita, H., et al. (2008). A novel stem cell source for vasculogenesis in ischemia: subfraction of side population cells from dental pulp. Stem Cells 26, 2408-2418. doi: 10.1634/ stemcells.2008-0393

Ishimatsu, H., Kitamura, C., Morotomi, T., Tabata, Y., Nishihara, T., Chen, K. K., et al. (2009). Formation of dentinal bridge on surface of regenerated dental pulp in dentin defects by controlled release of fibroblast growth factor- 2 from gelatin hydrogels. J. Endod. 35, 858-865. doi: 10.1016/j.joen.2009.03.049

Itoh, Y., Sasaki, J. I., Hashimoto, M., Katata, C., Hayashi, M., and Imazato, S. (2018). Pulp regeneration by 3-dimensional dental pulp stem cell constructs. J. Dent. Res. 97, 1137-1143. doi: 10.1177/0022034518772260

Jadhav, G., Shah, N., and Logani, A. (2012). Revascularization with and without platelet-rich plasma in nonvital, immature, anterior teeth: a pilot clinical study. J. Endod. 38, 1581-1587. doi: 10.1016/j.joen.2012.09.010

Jarmalavičiūtè, A., Tunaitis, V., Pivoraitè, U., Venalis, A., and Pivoriūnas, A. (2015). Exosomes from dental pulp stem cells rescue human dopaminergic neurons from 6-hydroxy-dopamine-induced apoptosis. Cytotherapy 17, 932939. doi: 10.1016/j.jcyt.2014.07.013

Kim, J. Y., Xin, X., Moioli, E. K., Chung, J., Lee, C. H., Chen, M., et al. (2010). Regeneration of dental-pulp-like tissue by chemotaxis-induced cell homing. Tissue Eng. Part A 16, 3023-3031. doi: 10.1089/ten.TEA.2010.0181

Lambrichts, I., Driesen, R. B., Dillen, Y., Gervois, P., Ratajczak, J., Vangansewinkel, T., et al. (2017). Dental pulp stem cells: their potential in reinnervation and angiogenesis by using scaffolds. J. Endod. 43, S12-S16. doi: 10.1016/j.joen.2017. 06.001

Li, J., Rao, Z., Zhao, Y., Xu, Y., Chen, L., Shen, Z., et al. (2020). A Decellularized matrix hydrogel derived from human dental pulp promotes dental pulp stem cell proliferation, migration, and induced multidirectional differentiation In Vitro. J. Endod. 46, 1438-1447.e5. doi: 10.1016/j.joen.2020. 07.008

Li, R., Guo, W., Yang, B., Guo, L., Sheng, L., Chen, G., et al. (2011). Human treated dentin matrix as a natural scaffold for complete human dentin tissue regeneration. Biomaterials 32, 4525-4538. doi: 10.1016/j.biomaterials.2011. 03.008

Linde, A. (1985). The extracellular matrix of the dental pulp and dentin. J. Dent. Res. 64, 523-529. doi: 10.1177/002203458506400405 
Liu, J. Y., Chen, X., Yue, L., Huang, G. T., and Zou, X. Y. (2015). CXC chemokine receptor 4 is expressed paravascularly in apical papilla and coordinates with stromal cell-derived factor- $1 \alpha$ during transmigration of stem cells from apical papilla. J. Endod. 41, 1430-1436. doi: 10.1016/j.joen.2015.04.006

Lu, J., Li, Z., Wu, X., Chen, Y., Yan, M., Ge, X., et al. (2019). iRoot BP Plus promotes osteo/odontogenic differentiation of bone marrow mesenchymal stem cells via MAPK pathways and autophagy. Stem Cell Res. Ther. 10:222. doi: 10.1186/ s13287-019-1345-3

Meng, H., Hu, L., Zhou, Y., Ge, Z., Wang, H., Wu, C. T., et al. (2020). A sandwich structure of human dental pulp stem cell sheet, treated dentin matrix, and matrigel for tooth root regeneration. Stem Cells Dev. 29, 521-532. doi: 10.1089/ scd.2019.0162

Mitsiadis, T. A., Magloire, H., and Pagella, P. (2017). Nerve growth factor signalling in pathology and regeneration of human teeth. Sci. Rep. 7:1327. doi: 10.1038/ s41598-017-01455-3

Miura, M., Gronthos, S., Zhao, M., Lu, B., Fisher, L. W., Robey, P. G., et al. (2003). SHED: stem cells from human exfoliated deciduous teeth. Proc. Natl. Acad. Sci. U.S.A. 100, 5807-5812. doi: 10.1073/pnas.0937635100

Morsczeck, C., and Reichert, T. E. (2018). Dental stem cells in tooth regeneration and repair in the future. Expert. Opin. Biol. Ther. 18, 187-196. doi: 10.1080/ 14712598.2018.1402004

Moussa, D. G., and Aparicio, C. (2019). Present and future of tissue engineering scaffolds for dentin-pulp complex regeneration. J. Tissue Eng. Regen. Med. 13, 58-75. doi: 10.1002/term.2769

Murakami, M., Horibe, H., Iohara, K., Hayashi, Y., Osako, Y., Takei, Y., et al. (2013). The use of granulocyte-colony stimulating factor induced mobilization for isolation of dental pulp stem cells with high regenerative potential. Biomaterials 34, 9036-9047. doi: 10.1016/j.biomaterials.2013.08.011

Na, S., Zhang, H., Huang, F., Wang, W., Ding, Y., Li, D., et al. (2016). Regeneration of dental pulp/dentine complex with a three-dimensional and scaffold-free stem-cell sheet-derived pellet. J. Tissue Eng. Regen. Med. 10, 261-270. doi: 10.1002/term.1686

Nagata, J. Y., Rocha-Lima, T. F., Gomes, B. P., Ferraz, C. C., Zaia, A. A., SouzaFilho, F. J., et al. (2015). Pulp revascularization for immature replanted teeth: a case report. Aust. Dent. J. 60, 416-420. doi: 10.1111/adj.12342

Nakashima, M., and Iohara, K. (2017). Recent progress in translation from bench to a pilot clinical study on total pulp regeneration. J. Endod. 43, S82-S86. doi: 10.1016/j.joen.2017.06.014

Nakashima, M., Iohara, K., Murakami, M., Nakamura, H., Sato, Y., Ariji, Y., et al. (2017). Pulp regeneration by transplantation of dental pulp stem cells in pulpitis: a pilot clinical study. Stem Cell Res. Ther. 8:61. doi: 10.1186/s13287017-0506-5

Pan, S., Dangaria, S., Gopinathan, G., Yan, X., Lu, X., Kolokythas, A., et al. (2013). SCF promotes dental pulp progenitor migration, neovascularization, and collagen remodeling - potential applications as a homing factor in dental pulp regeneration. Stem Cell Rev. Rep. 9, 655-667. doi: 10.1007/s12015-0139442-7

Park, S. Y., Sun, E. G., Lee, Y., Kim, M. S., Kim, J. H., Kim, W. J., et al. (2018). Autophagy induction plays a protective role against hypoxic stress in human dental pulp cells. J. Cell Biochem. 119, 1992-2002. doi: 10.1002/jcb.26360

Peng, C., Zhao, Y., Wang, W., Yang, Y., Qin, M., and Ge, L. (2017). Histologic findings of a human immature revascularized/regenerated tooth with symptomatic irreversible pulpitis. J. Endod. 43, 905-909. doi: 10.1016/j. joen.2017.01.031

Pivoraitè, U., Jarmalavičiūtè, A., Tunaitis, V., Ramanauskaitè, G., Vaitkuvienè, A., Kašèta, V., et al. (2015). Exosomes from human dental pulp stem cells suppress carrageenan-induced acute inflammation in mice. Inflammation 38, 1933-1941. doi: 10.1007/s10753-015-0173-6

Rahman, S. U., Oh, J. H., Cho, Y. D., Chung, S. H., Lee, G., Baek, J. H., et al. (2018). Fibrous topography-potentiated canonical wnt signaling directs the odontoblastic differentiation of dental pulp-derived stem cells. ACS Appl. Mater. Interfaces 10, 17526-17541. doi: 10.1021/acsami.7b 19782

Rosa, V., Zhang, Z., Grande, R. H., and Nör, J. E. (2013). Dental pulp tissue engineering in full-length human root canals. J. Dent. Res. 92, 970-975. doi: 10.1177/0022034513505772

Seo, B. M., Miura, M., Gronthos, S., Bartold, P. M., Batouli, S., Brahim, J., et al. (2004). Investigation of multipotent postnatal stem cells from human periodontal ligament. Lancet 364, 149-155. doi: 10.1016/s0140-6736(04) 16627-0

Shafiq, M., Jung, Y., and Kim, S. H. (2015). In situ vascular regeneration using substance P-immobilised poly(L-lactide-co- $\varepsilon$-caprolactone) scaffolds: stem cell recruitment, angiogenesis, and tissue regeneration. Eur. Cell Mater. 30, 282302. doi: 10.22203/ecm.v030a20

Shi, L., Fu, S., Fahim, S., Pan, S., Lina, H., Mu, X., et al. (2017). TNF-alpha stimulation increases dental pulp stem cell migration in vitro through integrin alpha-6 subunit upregulation. Arch. Oral. Biol. 75, 48-54. doi: 10.1016/j. archoralbio.2016.12.005

Sjöqvist, S., Ishikawa, T., Shimura, D., Kasai, Y., Imafuku, A., Bou-Ghannam, S., et al. (2019). Exosomes derived from clinical-grade oral mucosal epithelial cell sheets promote wound healing. J. Extracell Vesicles 8:1565264. doi: 10.1080/ 20013078.2019.1565264

Smith, J. G., Smith, A. J., Shelton, R. M., and Cooper, P. R. (2015). Dental pulp cell behavior in biomimetic environments. J. Dent. Res. 94, 1552-1559. doi: 10.1177/0022034515599767

Sonoyama, W., Liu, Y., Yamaza, T., Tuan, R. S., Wang, S., Shi, S., et al. (2008). Characterization of the apical papilla and its residing stem cells from human immature permanent teeth: a pilot study. J. Endod. 34, 166-171. doi: 10.1016/j. joen.2007.11.021

Sui, B., Chen, C., Kou, X., Li, B., Xuan, K., Shi, S., et al. (2019a). Pulp stem cellmediated functional pulp regeneration. J. Dent. Res. 98, 27-35. doi: 10.1177/ 0022034518808754

Sui, B. D., Zhu, B., Hu, C. H., Zhao, P., and Jin, Y. (2019b). Reconstruction of regenerative stem cell niche by cell aggregate engineering. Methods Mol. Biol. 2002, 87-99. doi: 10.1007/7651_2018_186

Sukho, P., Hesselink, J. W., Kops, N., Kirpensteijn, J., Verseijden, F., and Bastiaansen-Jenniskens, Y. M. (2018). Human mesenchymal stromal cell sheets induce macrophages predominantly to an anti-inflammatory phenotype. Stem Cells Dev. 27, 922-934. doi: 10.1089/scd.2017.0275

Suzuki, T., Lee, C. H., Chen, M., Zhao, W., Fu, S. Y., Qi, J. J., et al. (2011). Induced migration of dental pulp stem cells for in vivo pulp regeneration. J. Dent. Res. 90, 1013-1018. doi: 10.1177/0022034511408426

Tsai, A. C., Liu, Y., Yuan, X., and Ma, T. (2015). Compaction, fusion, and functional activation of three-dimensional human mesenchymal stem cell aggregate. Tissue Eng. Part A 21, 1705-1719. doi: 10.1089/ten.TEA.2014. 0314

Verma, P., Nosrat, A., Kim, J. R., Price, J. B., Wang, P., Bair, E., et al. (2017). Effect of residual bacteria on the outcome of pulp regeneration In Vivo. J. Dent. Res. 96, 100-106. doi: 10.1177/0022034516671499

Wigler, R., Kaufman, A. Y., Lin, S., Steinbock, N., Hazan-Molina, H., and Torneck, C. D. (2013). Revascularization: a treatment for permanent teeth with necrotic pulp and incomplete root development. J. Endod. 39, 319-326. doi: 10.1016/j. joen.2012.11.014

Xian, X., Gong, Q., Li, C., Guo, B., and Jiang, H. (2018). Exosomes with highly angiogenic potential for possible use in pulp regeneration. J. Endod. 44, 751758. doi: 10.1016/j.joen.2017.12.024

Xiao, L., Kumazawa, Y., and Okamura, H. (2014). Cell death, cavitation and spontaneous multi-differentiation of dental pulp stem cells-derived spheroids in vitro: a journey to survival and organogenesis. Biol. Cell 106, 405-419. doi: 10.1111/boc. 201400024

Xiao, M., Yao, B., Zhang, B. D., Bai, Y., Sui, W., Wang, W., et al. (2019). Stromalderived Factor- $1 \alpha$ signaling is involved in bone morphogenetic protein-2induced odontogenic differentiation of stem cells from apical papilla via the Smad and Erk signaling pathways. Exp. Cell Res. 381, 39-49. doi: 10.1016/j. yexcr.2019.04.036

Xiao, N., Yu, W. Y., and Liu, D. (2018). Glial cell-derived neurotrophic factor promotes dental pulp stem cell migration. J. Tissue Eng. Regen. Med. 12, 705-714. doi: 10.1002/term.2490

Xuan, K., Li, B., Guo, H., Sun, W., Kou, X., He, X., et al. (2018). Deciduous autologous tooth stem cells regenerate dental pulp after implantation into injured teeth. Sci. Transl. Med. 10:eaaf3227. doi: 10.1126/scitranslmed. aaf3227

Yang, B., Chen, G., Li, J., Zou, Q., Xie, D., Chen, Y., et al. (2012). Tooth root regeneration using dental follicle cell sheets in combination with a dentin matrix - based scaffold. Biomaterials 33, 2449-2461. doi: 10.1016/j.biomaterials. 2011.11.074 
Yang, J., Yuan, G., and Chen, Z. (2016). Pulp regeneration: current approaches and future challenges. Front. Physiol. 7:58. doi: 10.3389/fphys.2016.00058

Yang, J. W., Zhang, Y. F., Wan, C. Y., Sun, Z. Y., Nie, S., Jian, S. J., et al. (2015). Autophagy in SDF-1 $\alpha$-mediated DPSC migration and pulp regeneration. Biomaterials 44, 11-23. doi: 10.1016/j.biomaterials.2014.12.006

Yang, X., Ma, Y., Guo, W., Yang, B., and Tian, W. (2019). Stem cells from human exfoliated deciduous teeth as an alternative cell source in bio-root regeneration. Theranostics 9, 2694-2711. doi: 10.7150/thno.31801

Yavropoulou, M. P., and Yovos, J. G. (2016). The molecular basis of bone mechanotransduction. J. Musculoskelet Neuronal Interact 16, 221-236.

Yuan, H., Zhao, H., Wang, J., Zhang, H., Hong, L., Li, H., et al. (2018). MicroRNA let-7c-5p promotes osteogenic differentiation of dental pulp stem cells by inhibiting lipopolysaccharide-induced inflammation via HMGA2/PI3K/Akt signal blockade. Clin. Exp. Pharmacol. Physiol. 46, 389-397. doi: 10.1111/14401681.13059

Zhang, D. D., Chen, X., Bao, Z. F., Chen, M., Ding, Z. J., and Zhong, M. (2014). Histologic comparison between platelet-rich plasma and blood clot in regenerative endodontic treatment: an animal study. J. Endod. 40, 1388-1393. doi: 10.1016/j.joen.2014.03.020

Zhang, M., Jiang, F., Zhang, X., Wang, S., Jin, Y., Zhang, W., et al. (2017a). The effects of platelet-derived growth factor-bb on human dental pulp stem cells mediated dentin-pulp complex regeneration. Stem Cells Transl. Med. 6, 2126-2134. doi: 10.1002/sctm.17-0033

Zhang, Q., Nguyen, A. L., Shi, S., Hill, C., Wilder-Smith, P., Krasieva, T. B., et al. (2012). Three-dimensional spheroid culture of human gingiva-derived mesenchymal stem cells enhances mitigation of chemotherapy-induced oral mucositis. Stem Cells Dev. 21, 937-947. doi: 10.1089/scd.2011.0252

Zhang, S., Thiebes, A. L., Kreimendahl, F., Ruetten, S., Buhl, E. M., Wolf, M., et al. (2020a). Extracellular vesicles-loaded fibrin gel supports rapid neovascularization for dental pulp regeneration. Int. J. Mol. Sci. 21:4226. doi: 10.3390/ijms21124226
Zhang, S., Yang, Y., Jia, S., Chen, H., Duan, Y., Li, X., et al. (2020b). Exosomelike vesicles derived from Hertwig's epithelial root sheath cells promote the regeneration of dentin-pulp tissue. Theranostics 10, 5914-5931. doi: 10.7150/ thno. 43156

Zhang, W., Vazquez, B., Oreadi, D., and Yelick, P. C. (2017b). Decellularized tooth bud scaffolds for tooth regeneration. J. Dent. Res. 96, 516-523. doi: 10.1177/ 0022034516689082

Zhang, X., Li, H., Sun, J., Luo, X., Yang, H., Xie, L., et al. (2017c). Cellderived micro-environment helps dental pulp stem cells promote dental pulp regeneration. Cell Prolif. 50:e12361. doi: 10.1111/cpr. 12361

Zhao, H., Feng, J., Seidel, K., Shi, S., Klein, O., Sharpe, P., et al. (2014). Secretion of shh by a neurovascular bundle niche supports mesenchymal stem cell homeostasis in the adult mouse incisor. Cell Stem Cell 14, 160-173. doi: 10. 1016/j.stem.2013.12.013

Zhujiang, A., and Kim, S. G. (2016). Regenerative endodontic treatment of an immature necrotic molar with arrested root development by using recombinant human platelet-derived growth factor: a case report. J. Endod. 42, 72-75. doi: 10.1016/j.joen.2015.08.026

Conflict of Interest: The authors declare that the research was conducted in the absence of any commercial or financial relationships that could be construed as a potential conflict of interest.

Copyright (C) 2021 Huang, Li, Liu, Liu, Guo, Wu, Yang, Han and Xuan. This is an open-access article distributed under the terms of the Creative Commons Attribution License (CC BY). The use, distribution or reproduction in other forums is permitted, provided the original author(s) and the copyright owner(s) are credited and that the original publication in this journal is cited, in accordance with accepted academic practice. No use, distribution or reproduction is permitted which does not comply with these terms. 